\title{
A Commentary on Association of variants in genes involved in environmental chemical metabolism and risk of cryptorchidism and hypospadias
}

\author{
Maki Fukami \\ Journal of Human Genetics (2012) 57, 405-406; doi:10.1038/jhg.2012.76; published online 14 June 2012
}

$\mathrm{C}$ ryptorchidism (CO) and hypospadias (HS) are common congenital abnormalities that affect $\sim 1 \%$ of full-term male newborns. ${ }^{1-3} \mathrm{CO}$ and HS usually occur as multifactorial traits subject to a number of genetic and environmental factors with relatively minor effects, although in rare cases these conditions can occur as monogenic disorders because of mutations in genes such as $A R$ and SRD5A2.,3

During the last few decades, the prevalence of undermasculinized male genitalia, including $\mathrm{HS}$ and $\mathrm{CO}$, has increased in several countries. ${ }^{4}$ Similarly, compromised male reproductive function has recently been documented in various wildlife species. ${ }^{5}$ The rapid increase in the prevalence of undermasculinized genitalia indicates that intrauterine exposure to environmental chemicals with endocrine-disrupting properties (environmental endocrine disruptors (EEDs)) has a role in the development of such abnormalities, because large quantities of EEDs have been released into the environment in recent decades. ${ }^{5}$ Consistent with this notion, studies on laboratory animals have shown that intrauterine and lactational exposure to EEDs, such as 2,3,7,8-tetrachlorodibenzo- $p$-dioxin, affects the reproductive system of male offsprings. ${ }^{1,5}$

The effect of EEDs on male reproductive organs appears to depend on the susceptibility of the individual, in addition to the dosage and types of EEDs and the developmental stages of exposure. Till date, several association studies have been performed for

M Fukami is at the Department of Molecular Endocrinology, National Research Institute for Child Health and Development, Tokyo, Japan.

E-mail: mfukami@nch.go.jp patients with $\mathrm{CO}$ and HS, in order to identify genetic variants that predispose individuals to these conditions. ${ }^{2,3}$ Consequently, polymorphisms in several genes, such as $A R$, ESR1, ESR2 and SRD5A2, have been identified as the disease-associated variants. ${ }^{2,3}$ However, polymorphisms in genes involved in EED metabolism have been poorly investigated in patients with $\mathrm{CO}$ and $\mathrm{HS}$.

In this issue of the Journal, Qin et al. ${ }^{6}$ report an association between single-nucleotide polymorphisms (SNPs) in genes involved in EED metabolism and the risk of CO and HS. They examined 384 SNPs of 15 genes in 334 Japanese male subjects (95 patients with CO, 98 patients with HS and 141 unaffected controls) and 187 Italian male subjects (58 patients with $\mathrm{CO}$ and 129 controls), and identified SNPs in 5 genes that were over-represented in the patient group(s) in comparison with the control group. Notably, a significant association was found between SNPs in the aryl hydrocarbon receptor nuclear translocator 2 gene (ARNT2) and the risk of CO and HS in both the Japanese and Italian groups. Furthermore, a SNP in the aryl hydrocarbon receptor gene $(A H R)$ was found to be associated with $\mathrm{CO}$ in the Italian group. In this regard, it is noteworthy that both ARNT2 and AHR have been implicated in the dioxin signaling pathway., In the cytoplasm, dioxins bind to AHR and the ligand-bound AHR dimerizes with ARNT. The ligand-activated AHR-ARNT heterodimer then transactivates several target genes and modulates estrogen receptor signaling. ${ }^{1}$ ARNT2 has homology with ARNT and is predicted to interact with AHR. ${ }^{7}$ Thus, the results of this study imply that specific alleles of genes involved in the dioxin signaling pathway confer genetic susceptibility to EEDs, and thereby lead to undermasculinized genitalia. This notion is consistent with previous studies by Fujita et al. ${ }^{8}$ and Soneda et al. ${ }^{9}$ showing a significant association between the presence of micropenis, a phenotype of undermasculinization, and a SNP in AHRR encoding AHR repressor that is a component of a negative feedback loop in the dioxin-AHR signaling pathway.

Qin et al. ${ }^{6}$ found that SNPs in CYP1A2, NR1I2 and CYP17A1 are also associated with the risk of CO and/or HS. Of these, CYP1A2 and NR1I2 are involved in EED metabolism, and CYP17A1 encodes one of the key enzymes for estrogen biosynthesis. Interaction analysis for the positive SNPs indicated possible synergic interactions between SNPs in ARNT2 and those in the four other genes in the predisposition for CO or HS. ${ }^{6}$ These results indicate that complex gene-gene and gene-environmental interactions constitute risk factors for EED-induced genital abnormalities. Nevertheless, the mechanisms by which the SNPs identified in the present study increase the risk of genital abnormalities still need to be elucidated. These SNPs may serve only as markers for true hidden functional polymorphisms, because these are located in introns or the upstream regions of the corresponding gene and do not affect the amino-acid sequence.

Collectively, the study by Qin et al. provides additional information about the etiology of male genital abnormalities. As HS, $\mathrm{CO}$, testicular cancer and poor semen quality have recently been hypothesized to be symptoms of the same underlying entity (testicular dysgenesis syndrome), ${ }^{10}$ the SNPs associated 
with $\mathrm{CO}$ and HS may also be associated with other types of testicular dysgenesis syndrome. In fact, similar to the findings for $\mathrm{CO}$ and HS, a secular trend toward increased incidence has been reported for testicular cancer and spermatogenic failure. ${ }^{10}$ Thus, further association studies are necessary for genes involved in EED metabolism, particularly AHR, ARNT2, CYP1A2, CYP17A1 and NR1I2, for patients with testicular cancer and spermatogenic failure.

1 Virtanen, H. E. \& Adamsson, A. Cryptorchidism and endocrine disrupting chemicals. Mol. Cell Endocrinol. 355, 208-220 (2012).
2 Massart, F. \& Saggese, G. Morphogenetic targets and genetics of undescended testis. Sex. Dev. 4, 326-335 (2010).

3 van der Zanden, L. F., van Rooij, I. A., Feitz, W. F., Franke, B., Knoers, N. V. \& Roeleveld, N. Aetiology of hypospadias: a systematic review of genes and environment. Hum. Reprod. Update 8, 260-283 (2012).

4 Toppari, J., Kaleva, M. \& Virtanen, H. E. Trends in the incidence of cryptorchidism and hypospadias, and methodological limitations of registry-based data. Hum. Reprod. Update 7, 282-286 (2001).

5 Colborn, T., vom Saal, F. S. \& Soto, A. M. Developmental effects of endocrine-disrupting chemicals in wildlife and humans. Environ. Health Perspect. 101, 378-384 (1993).

6 Qin, X.-Y., Kojima, Y., Mizuno, K., Ueoka, K., Massart, F., Spinelli, C. et al. Association of variants in genes involved in environmental chemical metabolism and risk of cryptorchidism and hypospadias. J. Hum. Genet. 57, 434-441 (2012).
7 Hirose, K., Morita, M., Ema, M., Mimura, J., Hamada, H., Fujii, H. et al. cDNA cloning and tissue-specific expression of a novel basic helix-loop-helix/PAS factor (Arnt2) with close sequence similarity to the aryl hydrocarbon receptor nuclear translocator (Arnt). Mol. Cell. Biol. 16, 1706-1713 (1996).

8 Fujita, H., Kosaki, R., Yoshihashi, H., Ogata, T., Tomita, M., Hasegawa, T. et al. Characterization of the aryl hydrocarbon receptor repressor gene and association of its Pro185Ala polymorphism with micropenis. Teratology 65, 10-18 (2002).

9 Soneda, S., Fukami, M., Fujimoto, M., Hasegawa, T., Koitabashi, Y. \& Ogata, T. Association of micropenis with Pro185Ala polymorphism of the gene for aryl hydrocarbon receptor repressor involved in dioxin signaling. Endocr. J. 52, 83-88 (2005).

10 Toppari, J., Virtanen, H. E., Main, K. M. \& Skakkebaek N, E. Cryptorchidism and hypospadias as a sign of testicular dysgenesis syndrome (TDS): environmental connection. Birth Defects Res. A Clin. Mol. Teratol. 88, 910-919 (2010). 